\title{
Publisher Correction to: An exploration of subjective age, actual age, age awareness, and engagement in everyday
}

\author{
Joann M. Montepare ${ }^{1}$ \\ Published online: 23 October 2020 \\ (c) Springer Nature B.V. 2020
}

Keywords Subjective age $\cdot$ Age identification $\cdot$ Age attitudes
People's perceptions of their age have taken many forms, one of which is subjective age (Diehl et al. 2014). The construct of subjective age-which is the age people experience themselves to be-has been examined in different ways from various perspectives (Kastenbaum et al. 1972; Montepare 2009). For example, several different measures have been used, from measures of actual age estimates to scale responses, and from single items measures (e.g., what age do you feel) to more multidimensional measures (e.g., psychological, physical, social). As well, characterizations of subjective age have been investigated across diverse groups, including age groups ranging from adolescents to older adults, and cultural groups across the globe (e.g., USA, Germany, Senegal, Japan). Taken together, findings have shown an interesting and persistent pattern. Specifically, whereas younger individuals typically feel somewhat older than their age, individuals begin to feel younger than their age between 20 and 30 years, with a younger discrepancy increasing with advancing age. In addition to this overall life span pattern, there is a good deal of variability along the age continuum

Responsible editor: Matthias Kliegel.

For reason beyond the control of the editors, the article titled "An exploration of subjective age, actual age, age awareness, and engagement in everyday behaviors" by Joann M. Montepare (https ://doi.org/10.1007/s10433-019-00534-w) was published in the regular issue Vol. 17 issue 3 instead of this special issue, where it was originally scheduled to appear. Therefore, the full article is reprinted here.

The original article can be found online at https://doi.org/10.1007/ s10433-019-00534-w.

Joann M. Montepare

jmontepare@lasell.edu

1 RoseMary B. Fuss Center for Research on Aging and Intergenerational Studies, Lasell University, 1844

Commonwealth Avenue, Newton, MA 02466, USA as well as across time and circumstance (Barrett and Montepare 2015; Galambos et al. 2005; Hatta et al. 2010; Macia et al. 2012; Westerhof et al. 2003). Adding to this variability, psychological factors such as the extent to which individuals consider age to be a defining personal attribute-described as age awareness-have been implicated in the subjective age individuals experience themselves to be (Montepare 2009).

An equally interesting, and perhaps more significant observation, is that subjective age often rivals or exceeds actual age as a predictor of other perceptions associated with health and well-being (Montepare 2009; Wurm et al. 2017; Westerhof et al. 2014). Within this realm, research has documented relationships between subjective age and a variety of consequential outcomes, especially in later adulthood. For example, experimental studies have evidenced the transient effects of subjective age on vision, memory, and health outcomes, whereas observational longitudinal studies have shown longer-term links to outcomes such as stress, physical health, cognitive functioning, and even longevity and mortality (Bellingtier et al. 2017; Kornadt et al. 2018; Stephan et al. 2015; Westerhof et al. 2014; Westerhof and Wurm 2015; Wurm et al. 2017). Cross-sectional studies have also demonstrated consequences of subjective age. For example, comparisons of magnetic resonance imaging (MRI) scans of healthy adults have shown differences across groups of older adults who differed in the extent to which they felt younger, the same, or older than their actual age. Adults with younger identities had greater regional gray matter volumes as well as younger predicted brain age, outcomes with implications for later life neurocognitive health (Kwak et al. 2018).

Although ample empirical data have provided revealing information about the nature and implications of subjective age, important questions remain about the dynamics of age identification and its consequences. Moreover, understanding these dynamics is important because personal 
perceptions of age are often more predictive of physical, psychological, and social outcomes than are other factors, such as gender, education level, or physical status (Wurm et al. 2017). The present study explored these dynamics within the realm of everyday behaviors, along with the extent to which subjective age predicted engagement in daily behaviors in comparison with the related factors of actual age and age awareness. To understand the motivation for a focus on everyday behaviors, theoretical considerations are presented as background.

Contemporary scholars have made the case that views on aging function by way of proximal and distal biologicalevolutionary, psychological, and social-contextual factors (Kornadt et al. 2019). Several models within this framework have been offered to explain how subjective age drives the outcomes that have been observed in existing empirical investigations (Barrett and Montepare 2015; Diehl et al. 2015; Wurm et al. 2017). Of particular interest for the present study, Levy (2009) proposed three possible pathways by which age perceptions exert their influence. A physiological pathway implicates psychological stress associated with negative perceptions of age that results in an elevated physiological stress response which shapes negative outcomes, especially adverse health effects. A psychological pathway operates by way of self-fulfilling prophecy mechanisms and motivational factors whereby negative age expectations become integrated into negative self-views and yield related negative outcomes. A behavioral pathway assumes that adults with more positive perceptions of age engage in more positive and health-promoting behaviors, whereas those with negative perceptions engage in negative and unhealthy practices, or fail to behave in positive activities because negative perceptions undermine their psychological resources (Wurm and Schüz 2015).

Although informative, the bulk of work linking various pathways to various outcomes associated with perceptions of age has focused mainly on the implications of the negative age stereotypes held by adults as opposed to the subjective age they experience themselves to be (Wurm et al. 2017). However, there is evidence to suggest that outcomes associated with subjective age are shaped by similar pathways. Consistent with a physiological pathway, Stephan and colleagues showed that a younger subjective age in older adults was associated with lower C-reactive protein (a marker of systemic inflammation associated with stress) in data from the Health and Retirement Study (Stephan et al. 2015). As well, Bellingtier et al. (2017) found that subjective age was associated with daily stressors such that on days when people experienced more than average health problems, stress, or negative affect, they felt older than on days when average health, stress, and negative affect were experienced.

In line with psychological mechanisms, Hughes and Lachman (2018) examined subjective age and social comparisons in a sample of adults in the Midlife in the US (MIDUS) study on two occasions 10 years apart. Their analyses showed that those with younger subjective ages who did not feel that they had aged by much had more favorable social comparative assessments, as well as better health and memory, than those who held older identities. Behavioral pathways have also been implicated in outcomes associated with subjective age. For instance, longitudinal research found that a younger subjective age was associated with faster walking and a lower decline in walking speed over time, which is a predictor of health in old age (Stephan et al. 2015). Using MIDUS data, Stephan and colleagues demonstrated that a younger subjective age was linked with better cognitive functioning 10 years later and was partially mediated by individuals' level of physical activity (Stephan et al. 2016). Others have also found links between subjective age and behaviors such as exercise, cessation of driving, sleep, and clothes shopping (Montepare and Conte 2014; Pachana et al. 2017; Stephan et al. 2017; Wienert et al. 2015).

Personal perceptions of age have been a focus of lifespan social-developmental research since Kastenbaum et al. (1972) argued that how old people see themselves serves as a functional component of the self that can create and modify "old behavior." Clearly, empirical investigations have revealed much about the components and consequences of subjective age, and particular theoretical approaches have suggested possible routes by which one's age identity is linked to various outcomes. At the same time, there is still much to be learned, especially with respect to the behavioral pathways along which subjective age effects unfold. Behavioral pathways may be an especially important channel to elucidate for several reasons. For one thing, it may be argued that behaviors are ultimately a linchpin in other pathways. For example, while the psychological pathway may call attention to motivational processes that impact defensive self-protective reactions of the aging self (associated with younger age identities), such reactions at some point must involve behavioral reactions (such as health-defeating or health-promoting activities) that shape and reinforce outcomes. Intersections of pathways are further highlighted by process-oriented studies such as research by Wienert et al. (2017) who found that feeling physically younger was associated with higher planning to adopt higher levels of physical activity and more planning was associated with more subsequent physical activity.

A noticeable gap in the scant research that has explored behavioral routes is that this work has more often been designed around a particular behavior than an interest in varied or distinctive behavioral pathways. As such, this work has focused on singular, isolated behaviors, such as physical activity, rather than a range of daily behaviors in which people are likely to engage. If research is to move beyond addressing theoretical underpinnings and move toward 
behavioral interventions to enhance health and well-being, then a broader view of behavioral implications is needed. In addition, the few studies that have explored behavioral links associated with subjective age have most often used unidimensional measures of age identity. Relying on a unidimensional approach is limited for both conceptual and empirical reasons, and researchers have emphasized the merit of taking the multidimensional nature of subjective aging measures into account (Diehl et al. 2014; Spuling 2019). Assessing subjective aging in a unidimensional way ignores insights into the multidimensionality and multidirectionality of lifespan development, and evidence that multiple criteria are necessary to understand age-related outcomes. Furthermore, possible differential relations between dimensions of age identity and major developmental outcomes, such as well-being, health, or mortality, cannot be addressed with sufficient detail if only unidimensional scales are utilized. More generally, the present study approach is consistent with the current perspective that views on aging are best conceptualized as multidimensional, multidirectional, and multifunctional constructs (Kornadt et al. 2019).

It is also of concern that studies of subjective age may reflect an implicit bias that assumes that more youthful identities entail more positive behaviors and outcomes, which may not always be the case (Barrett and Montepare 2015). For example, Pachana et al. (2017) showed that older adults who had either ceased driving or had a plan to stop driving in the near future reported subjectively feeling older identities associated with these behavioral decisions. Similarly, Barrett and Gumber (2019) found that older (or less youthful) identities predicted future self-regulated driving behaviors and raised the suggestion that younger age identities may not always be in the best interest of individuals. Thus, whereas younger subjective ages may be associated with purported positive personal views and outcomes, they may also have a downside to the extent that they prompt individuals to resist some activities which may be considered of benefit or utility from aging perspective. One way to avoid such a bias is to build research around a range of everyday behaviors that may be both prompted and avoided in light of older adults' subjective age.

In examining relationships between subjective age and everyday behaviors, it is also important to consider psychological factors that may intersect with these connections. One factor of interest is the extent to which individuals consider age to be a defining personal attribute. Such age awareness has been shown to impact the extent to which individuals process self-relevant age-related information (Montepare and Clements 2001). It has also been shown to moderate relationships between subjective age and other self-perceptions. For example, women's older age identities were shown to have a strong relationship to negative views of their bodies, and this was especially true for women with higher age awareness who viewed their age to be an important personal attribute (Montepare 2014). Similarly, in a study of women's fashion behavior, not only were women with higher age awareness more likely to have older age identities in connection with more negative body esteem, they also reported being more fashion conscious and having more challenges when shopping for clothing than women for whom age was not a salient personal attribute (Montepare and Conte 2014). To gain a fuller picture of the relationship between subjective age and everyday behaviors, the potential moderating impact of individual's age awareness was also explored.

The goal of the present research was to explore the extent to which dimensions of people's subjective age were associated with how often they engaged in a range of everyday behaviors conceptually connected to health and wellbeing, as a way to begin to elucidate behavioral pathways. In an effort to offer a more fine-grained picture of possible implications of subjective age, the frequency with which people engaged in common day-to-day behaviors was also explored, in addition to the extent to which people's actual age, and their age awareness figured into these behavioral connections.

\section{Method}

\section{Sample}

SurveyMonkey (SurveyMonkey, San Mateo, CA, U.S.A.; http://www.surveymonkey.com) was used to create and administer an online survey to the target audience of older adults. Respondents included 196 adults (105 women and 91 men) ranging in age from 55 to 87 years $(M=66.05$ years, $\mathrm{SD}=7.7$ ). Approximately $20 \%$ of respondents has some college experience, $29 \%$ reported hold a bachelor's degree and about $37 \%$ indicated that they had graduate degree experience. The majority of participants identified themselves as White (92\%).

\section{Measures}

Respondents were first asked background questions about their gender, race, and educational level in addition to being asked to indicate their actual age in years. Seven-point scales, ranging from (1) a lot younger than my age to (7) a lot older than my age, were used to assess respondents' subjective age along four dimensions. The Likert-scale format was used in place of another commonly used subjective age format (i.e., numeric estimates in years used to derive discrepancy scores) for several reasons. Specifically, this format is better suited to assessing subjective age across age groups that differ widely in age. As Montepare (1996) has argued, while numeric estimates may allow for more specificity, 
they may also be more ambiguous to interpret. For example, given the nonequivalent nature of developmental time intervals, numeric discrepancies younger adults experience may appear smaller than those experienced by older adults, but the psychological meaning of the discrepancies may nevertheless be similar. Moreover, both measures have been found to be highly correlated when used within age groups. Thus, with an eye toward future research regarding everyday behaviors in younger age groups, the decision was made to use a scale format.

General felt age was assessed by asking respondents the age, in general, they felt. Additional subjective age scales reflecting abbreviated versions of the longer subjective age subscales of the SAGS (Subjective Age and Gender Scale, Montepare 1996) assessed more specific dimensions of subjective age. Psychological age was assessed by asking respondents the age they felt with respect to their general state of mind (e.g., abilities and motivations to learn things). Physical age was assessed by asking them the age they felt with respect to their physical condition and characteristics. Social age was assessed by asking respondents to indicate that age they felt with respect to their social activities and behaviors. In additional to subjective age, respondents' age awareness was assessed by asking if age was the first thing that came to mind when they thought about themselves. Responses were made on a 7-point scale ranging from (1) definitely no to (7) definitely yes.

A list of 29 behaviors was generated by the investigator and a team of two student interns. Behaviors reflecting everyday routine actions likely to be encountered by individuals in the sample age group and that could be seen as conceptually contributing to health and well-being were included in the list, as well as several other more formal official behaviors. Although not meant to be comprehensive, the list reflected a wide range of common behaviors about which individuals could easily respond in a survey format without feeling taxed (in order to avoid response bias). The extent to which individuals engaged in each behavior was assessed by asking them how often they did each activity using a 7-point scale with responses labeled (1) "have never done it", "very rarely", "rarely", "occasionally", "very frequently", and (7) "always do it". The specific behaviors included the following activities: floss my teeth, get an annual physical checkup, review retirement savings, review long-term health insurance, take a vacation, get a medical screening procedure (e.g., mammogram, colonoscopy), get a manicure or pedicure, go to a hair salon or barber, take a daily walk, go to the gym, take vitamins or other health supplements, visit with friends, review my will, weigh myself, learn a new skill (e.g., digital photography, cake decorating), do yoga or some form of meditation, get enough sleep, interact with people from a younger generation, interact with people from an older generation, engage in a religious service or prayer, have an eye exam, get my hearing checked, get my blood pressure or cholesterol level checked, shop for new clothes, use social media (e.g., Facebook, Twitter), participate in a senior citizen group, do a brain exercise (e.g., cross-word puzzle, online game), work overtime, and visit with family.

\section{Results}

\section{Preliminary analyses}

A principle components factor analysis with varimax rotation was conducted to examine the extent to which the individual behaviors could be combined into more parsimonious groupings for data analysis. Nine groups were identified, and composite scores were computed for each by averaging across individual ratings (see Table 1). These composites were used in all subsequent analyses. The first group was labeled Medical and included behaviors that dealt with getting an annual checkup, eye exam, tests for blood pressure and cholesterol, and taking vitamins. The Retirement group reflected behaviors that dealt with the review of one's savings, long-term health, and will. The Younger Social group included behaviors that reflecting taking a vacation, visiting with friends and family, and interacting with younger generations. The group labeled Older Social included the extent to which individuals interacted with older generations, participated in a senior citizen group and attended religious services. The Personal group included behaviors that dealt with going to the gym, doing yoga and meditation, and dental flossing. The Grooming group included behaviors that dealt with getting a manicure, a pedicure, and enough sleep. The Brain group reflected behaviors that dealt with learning a new skill, doing brain exercise, and working overtime, and the Body group captured behaviors that dealt with engaging in a daily walk and weight check. Finally, the group labeled Style included behaviors reflecting the extent to which an individual went to a hair salon or barber, shopped for new clothes, and used social media.

\section{Main analyses}

Correlational statistics are presented in Table 2. Initial zero-order correlational analyses showed that actual age was associated with several of the behavior groups. In particular, older respondents indicated that they were more likely to engage in Medical, Retirement, and Younger Social activities, but less likely to engage in Grooming and Body activities. Partial correlations between subjective age and the behavior groups also showed a number of significant associations. In these analyses, actual age was controlled in order to assess the independent effects of subjective age. Consistent with the view that subjective age is a multidimensional 
Table 1 Behavior groups derived from principal component analysis

\begin{tabular}{|c|c|c|c|c|c|c|c|c|c|}
\hline & \multirow[t]{2}{*}{ Medical } & \multirow[t]{2}{*}{ Retirement } & \multicolumn{7}{|l|}{ Behavior groups } \\
\hline & & & Younger social & Older social & Personal & Grooming & Brain & Body & Style \\
\hline Go to the gym & & & & & .756 & & & & \\
\hline Floss my teeth & & & & & .457 & & & & \\
\hline Do yoga or some form of meditation & & & & & .737 & & & & \\
\hline Get an annual physical checkup & .737 & & & & & & & & \\
\hline $\begin{array}{l}\text { Get a medical screening procedure (e.g., mam- } \\
\text { mogram, colonoscopy) }\end{array}$ & .800 & & & & & & & & \\
\hline Have an eye exam & .774 & & & & & & & & \\
\hline Get my hearing checked & .329 & & & & & & & & \\
\hline $\begin{array}{l}\text { Get my blood pressure or cholesterol level } \\
\text { checked }\end{array}$ & .801 & & & & & & & & \\
\hline Take vitamins or other health supplements & .420 & & & & & & & & \\
\hline Review my will & & .771 & & & & & & & \\
\hline Review retirement savings & & 609 & & & & & & & \\
\hline Review long-term health insurance & & 694 & & & & & & & \\
\hline Take a vacation & & & .719 & & & & & & \\
\hline Visit with friends & & & 617 & & & & & & \\
\hline Interact with people from a younger generation & & & 612 & & & & & & \\
\hline Visit with family & & & .737 & & & & & & \\
\hline Get a manicure or pedicure & & & & & & .348 & & & \\
\hline Get enough sleep & & & & & & .744 & & & \\
\hline Take a daily walk & & & & & & & & .663 & \\
\hline Weigh myself & & & & & & & & .363 & \\
\hline $\begin{array}{l}\text { Learn a new skill (e.g., digital photography, } \\
\text { cake decorating) }\end{array}$ & & & & & & & .537 & & \\
\hline $\begin{array}{l}\text { Do a brain exercise (e.g., cross-word puzzle, } \\
\text { online game) }\end{array}$ & & & & & & & 676 & & \\
\hline Work overtime & & & & & & & 622 & & \\
\hline Interact with people from an older generation & & & & .570 & & & & & \\
\hline Engage in religious service or prayer & & & & 681 & & & & & \\
\hline Participate in a senior citizen group & & & & .319 & & & & & \\
\hline Go to a hair salon or barber & & & & & & & & & 637 \\
\hline Shop for new clothes & & & & & & & & & .403 \\
\hline Use social media (e.g., Facebook, Twitter) & & & & & & & & & .510 \\
\hline
\end{tabular}

Values reflect the highest factor loadings for each of the behaviors listed

construct (Diehl et al. 2015; Montepare 2009), it was further observed that different aspects of subjective age were associated with somewhat different behavior groups. More specifically, people who held younger psychological, physical, and social subjective ages were more likely to engage in Personal and Grooming behaviors. People who held younger psychological ages were also more likely to engage in Brain behaviors, whereas people who reported younger social and physical subjective ages were more likely to engage in Younger Social and Style behaviors.

Although different aspects of subjective age showed somewhat varied patterns of association with different everyday behaviors, it is also of interest to note that the individual measures of subjective age were significantly correlated with each other (all $p s<.001$ ), with the general felt age measure being most highly correlated with measures of how old individuals experienced themselves to be psychologically, physical, and socially (see Table 3 ). To examine in broader detail the relationship of subjective age to everyday behaviors, a composite index of subjective age averaged across the individual measures was computed and labeled age identity. This measure was also significantly correlated with the singular felt age measure, and partial correlations with the everyday behavior group composites captured the overall patterns observed with the individual measures (see Table 2). That is, individuals with overall younger age identities reported that they engaged more frequently in behaviors in the Younger Social, Personal, Brain, Grooming, Body, and Style groups. 
Table 2 Correlations between behavior groups and actual age, subjective age, and age awareness

\begin{tabular}{lcllllll}
\hline Behavior group & Actual age & Felt age & Psychological age & Physical age & Social age & Age identity & Age awareness \\
\hline Medical & $.21^{* *}$ & -.05 & .01 & -.01 & -.08 & -.03 & $-.16^{*}$ \\
Younger social & $.15^{*}$ & $-.21^{* *}$ & -.11 & $-.19^{* *}$ & $-.16^{*}$ & $-.20^{*}$ & $-.17^{*}$ \\
Retirement & $.17^{*}$ & -.05 & -.08 & $-.16^{*}$ & -.04 & -.10 & $-.15^{*}$ \\
Personal & .02 & $-.21^{* *}$ & $-.24^{* *}$ & $-.27^{* *}$ & $-.30^{* *}$ & $-.32^{* *}$ & $-.14^{*}$ \\
Brain & -.05 & $-.18^{* *}$ & $-.15^{*}$ & -.09 & .01 & $-.14^{*}$ & -.06 \\
Grooming & $-.15^{*}$ & $-.25^{* *}$ & $-.16^{*}$ & $-.27^{* *}$ & $-.29^{* *}$ & $-.30^{* *}$ & -.06 \\
Older SOCIAL & .09 & -.02 & -.04 & -.02 & -.13 & -.08 & .01 \\
Body & $-.14^{*}$ & -.13 & -.06 & $-.26^{* *}$ & -.10 & $-.16^{*}$ & -.07 \\
Style & .05 & $-.17^{* *}$ & -.05 & $-.14^{*}$ & $-.17^{*}$ & $-.15^{*}$ & -.01 \\
\hline
\end{tabular}

Coefficients with actual age reflect zero-order correlations, all others reflect partial correlations controlling for actual age

$* p<.05 ; * * p<.01$

Table 3 Correlations among measures of subjective age

\begin{tabular}{llllll}
\hline & Felt age & Psychological age & Physical age & Social age & Age identity \\
\hline Actual age & $-.13+$ & $-.14+$ & -.07 & -.06 & $-.12+$ \\
Felt age & & $.71^{* *}$ & $.58^{* *}$ & $.51^{* *}$ & $.86^{* *}$ \\
Psychological age & & & $.53^{* *}$ & $.44^{* *}$ & $.81^{* *}$ \\
Physical age & & & & $.46^{* *}$ & $.82^{* *}$ \\
Social age & & & & & $.74^{* *}$ \\
\hline
\end{tabular}

$+p<.10 ; * * p<.01$

Correlations between age awareness and behavior group composites, controlling for actual age were also computed. As shown in Table 2, these analyses showed that individuals who reported greater age awareness were less likely to engage in Medical, Retirement, Younger Social, and Personal behaviors.

Regression analyses were conducted to examine the relative strength of actual age, age identity, and age awareness in predicting the frequency with which individuals reported engaging in each group of everyday behaviors. In these analyses, the possible interaction of age identity with age awareness was also assessed as a predictor of behaviors to examine if age awareness moderated any effects of age identity. Age identity and age awareness were significantly correlated $(r(194)=.26 . p<.001)$. As well, age identity was marginally correlated with actual

Table 4 Results of regression analyses predicting behavior group composites

\begin{tabular}{|c|c|c|c|c|c|c|c|c|c|}
\hline & Medical & Younger social & Retirement & Personal & Brain & Grooming & Older social & Body & Style \\
\hline \multicolumn{10}{|c|}{ Actual age } \\
\hline$\beta$ & .22 & .14 & .17 & -.01 & -.06 & .11 & .08 & .12 & .02 \\
\hline$t$ & $3.09 *$ & $1.95^{*}$ & $2.33^{*}$ & -.18 & -.76 & 1.51 & 1.16 & 1.62 & .33 \\
\hline \multicolumn{10}{|c|}{ Age identity } \\
\hline$\beta$ & .02 & -.16 & -.06 & -.3 & .12 & -.31 & -.08 & -.16 & -.17 \\
\hline$t$ & .28 & $-2.15^{*}$ & -.79 & $-4.10 * *$ & -1.56 & $-4.23 * *$ & -1.03 & $-2.08^{*}$ & $-2.18^{*}$ \\
\hline \multicolumn{10}{|c|}{ Age awareness } \\
\hline$\beta$ & -.17 & -.12 & -.14 & -.05 & -.04 & .03 & .02 & -.01 & .04 \\
\hline$t$ & $-2.34^{*}$ & -1.67 & -1.87 & -.75 & -.52 & .43 & .26 & -.17 & .53 \\
\hline \multicolumn{10}{|c|}{ Interaction age identity-age awareness } \\
\hline$\beta$ & -.03 & -.36 & -.04 & -.02 & -.02 & .07 & -.05 & .01 & .02 \\
\hline$t$ & -.40 & -.36 & -.55 & -.27 & -.31 & .96 & -.61 & .01 & .29 \\
\hline
\end{tabular}

$* p<.05 ; * * p<.01$ 
age $(r(194)=-.12, p=.10)$ although age awareness was not $(r(194)=.05, p=.46)$. Interaction terms for age identify and age awareness using standardized coefficients were computed and entered into regression analyses for each behavior group along with the other predictors. As seen in Table 4, subjective age emerged as the strongest and most consistent predictor across the behavior groups, especially those reflecting Younger Social, Personal, Grooming, Body, and Style behaviors The interaction of age identity and age awareness did not emerge as a significant predictor in any of these analyses (all ps $>.05$ ), indicating that age awareness did not moderate the observed associations between age identity and behaviors. Finally, actual age emerged as a significant predictor of behaviors in the Retirement group, as well as the Medical group for which age awareness was also a significant predictor.

\section{Discussion}

The present study sought to explore links between various perceptions of age and the frequency with which individuals engaged in a range of everyday behaviors. Several interesting patterns were observed in this sample of adults aged 55 and older. First, actual age predicted several particular behaviors. That is, individuals' advancing age was most strongly associated with the extent to which they reported getting annual checkups, having various physical examinations, and taking vitamins and other health supplements. With age, individuals were also more likely to review their retirement savings, will, and health insurance.

Individuals subjective age also was associated with several everyday behaviors, and it emerged as a stronger and more consistent predictor of behaviors than either individuals' actual age or their age awareness. Moreover, although different dimensions of subjective age were associated with various domains of behavior, supporting the notion that subjective age is a multidimensional construct (Diehl et al. 2014; Montepare 2009), they were highly intercorrelated and reflected in individuals' general age identity which significantly predicted a range of behaviors. More specifically, individuals who had younger age identities reported more often engaging in behaviors such as going on daily walks, to the gym, hair salon or barber, as well as shopping for new clothes, getting a manicure or pedicure, flossing, doing yoga or meditation, and getting enough sleep. They also more frequently reported taking vacations, visiting with family and friends, and interacting with younger generations. Moreover, engagement in these activities was not moderated by the extent to which individuals saw their age as a central personal attribute. However, individuals who had higher age awareness were less likely to engage in a few types of everyday behaviors.
In particular, they were less likely to engage in medically related behaviors, and to some extent retirement activities, as well as personal activities like going to the gym or meditating.

What do these findings suggest about the broader implications of perceptions of age and everyday behaviors? The present findings may be taken to indicate that some everyday behaviors are driven by age-related conditions and concerns. For example, engaging in medically related behaviors may reflect changes in physical condition that are more likely to be of increasing concern at older ages. Moreover, getting retirement planning and related undertakings in order may be tied to a person's actual age because such behaviors are linked one's career stage and concerns for economic security in later life. On the other hand, some everyday behaviors may be more aligned with how old individuals subjectively experience themselves to be because those behaviors are associated with more "youth-sustaining" motivations that fuel a desire to stay healthy and in shape, look attractive, and maintain social connections. However, individuals who are highly attuned to age may engage less in, or actively avoid, other behaviors like going to the doctor and seeking medical attention because these behaviors make one's age even more salient and elicit aging fears and stigma.

While the present study was successful in revealing links between perceptions of age and everyday behaviors, there is more to understand about the nature and dynamics of these relationships. First, one needs to question the extent to which self-reports of everyday behaviors such as those studied here are accurate and reliable. To this end, other methods of behavioral assessment such as daily diary studies would be useful in validating the observed relationships [see work by Bellingtier et al. (2017)].

It would also be of value to expand attention to other types of behaviors. For example, it would be useful to examine the extent to which particular health risk behaviors such as smoking, overeating, and alcohol, and drug consumption are associated with perceptions of age, especially if research in this realm is to inform behavioral intervention efforts. Arbeau et al. (2007) found that dating, sexual, and substance use activities figured importantly in younger individuals' older subjective age identities, and it would be useful to identify how these behavioral links play out across the life span. Similarly, behaviors related to media use, educational engagement, and work patterns would be of interest to examine across different age, gender, and social groups for a more complete mapping of everyday behaviors and perceptions of age. Attention to such behaviors is further motivated by research, indicating that engaging in activities such as social media use can have a range of positive consequences for older adults, and much can be gained from understanding how individuals' age identities fit into outcomes such as this (Leist 2013). 
With an understanding of associations between perceptions of age and everyday behaviors, a more process-oriented understanding of the dynamics of subjective age is closer at hand. However, several issues will need to be resolved. In particular, the issue of directionality remains a recognized, yet not well understood consideration. That is, the extent to which, or under what conditions, the age individuals experience themselves to be leads to engaging in certain behaviors versus engaging in particular behaviors shapes subjective age. Working out directionality will also challenge theorists to consider in more detail how psychological, behavioral, and physiological pathways intersect. While it may be the case that behavioral pathways are a core linchpin in a multipath model, more needs to be done to understand how they may drive other routes, along with potential mediating and moderating factors. The present research focused on only several among many age constructs which have been found to be of consequence to health and well-being across the life span, including self-perceptions of aging, attitudes toward aging, age stereotypes, and awareness of aging (Diehl et al. 2014; Kornadt et al. 2019). Thus, more needs to be done to examine how these different perceptions of age interrelate, as well as intersect, with everyday behaviors.

Certainly, research also is needed to examine how behavior links may vary across social groups that vary by gender, race, ethnicity, and socioeconomic background given that patterns and types of everyday behaviors will likely differ in these groups. Furthermore, such considerations call for the need to question how related sociological processes and structures also shape individuals' age perceptions and their behavioral activities (Barrett and Montepare 2015). For example, how might elements of our social structure that reflect systems of inequality (e.g., gender, race, ethnicity) along with social institutions (e.g., education, marriage, work, medicine) and social networks (e.g., family ties, social circles) factor into the behavioral opportunities and decisions individuals are likely to encounter across the life course? A life course perspective also calls for the need to consider how particular historical contexts might shape decisions about engaging in various behaviors across the life course. For example, given generational shifts in age demographics, the extension of life expectancy, the postponement of retirement from work, and later transitions to marriage and parenthood, individuals in recent (and future) cohorts may view some behavioral decisions and their implications differently. As well, the life course principle of "linked lives" highlights the importance of considering how relationships with significant others and friends may impact individuals' age perceptions and their behavioral choices (Barrett 2005).

Along these broader lines, the study of perceptions of age must ultimately consider the surrounding context in which behaviors are manifested, or even possible. It has become increasing clear that geography and characteristics of one's immediate neighborhood have a significant impact on health and well-being beyond individuals' personal views or motivations, owing in part to the ability of people to engage in particular kinds of behaviors (Dannenberg et al. 2011). Thus, the close study of perceptions of age and everyday behaviors at some point will need to step back and use a larger lens to examine the context in which individuals live, behave, and age. This will be of particular consequence for theorists with an eye toward translating research findings to age-friendly behavioral intervention strategies in communities.

\section{References}

Arbeau KJ, Galambos NL, Jansson SM (2007) Dating, sex, and substance use as correlates of adolescents' subjective experience of age. J Adolesc 30:435-447. https://doi.org/10.1177/0165025408 095552

Barrett A (2005) Gendered experiences in midlife: implications for age identity. J Aging Stud 19:163-183. https://doi.org/10.1016/j. jaging.2004.05.002B

Barrett A, Gumber C (2019) Feeling older and driving less: the effect of age identity on older adults' transition from driving. Innov Aging. https://doi.org/10.1093/geroni/igz004

Barrett A, Montepare JM (2015) "It's about time": applying life span and life course perspectives into the study of subjective age. Annu Rev Geriatr Gerontol 35:55-78. https://doi. org/10.1891/0198-8794.35.55

Bellingtier JA, Neupert SD, Kotter-Grühn D (2017) The combined effects of daily stressors and major life events on daily subjective ages. J Gerontol B Psychol Sci Soc Sci 72:613-621. https://doi. org/10.1093/geronb/gbv101

Dannenberg AL, Frumkin H, Jackson R (2011) Making healthy places: designing and building for health, well-being, and sustainability. Island Press, Washington

Diehl M, Wahl HW, Barrett AE, Brothers AF, Miche M, Montepare JM, Wurm S (2014) Awareness of aging: theoretical considerations on an emerging concept. Dev Rev 34:93-113. https://doi. org/10.1016/j.dr.2014.01.001

Diehl M, Wahl HW, Brothers A, Miche M (2015) Subjective aging and awareness of aging: toward a new understanding of the aging self. Annu Rev Gerontol Geriatr 35:1-28. https://doi. org/10.1891/0198-8794.35.1

Galambos NL, Turner PK, Tilton-Weaver LC (2005) Actual and subjective age in emerging adulthood: the crossover effect. J Adolesc Res 20:538-556. https://doi.org/10.1177/0743558405274876

Hatta T, Higashikawa M, Hatt T (2010) Subjective age in a modern Japanese young, middle-age, and upper middle-age sample. Percept Mot Skills 111:285-290. https://doi.org/10.2466/02.07.12.13.17. PMS.111.4.285-290

Hughes ML, Lachman ME (2018) Social comparisons of health and cognitive functioning contribute to changes in subjective age. J Gerontol B Psychol Sci Soc Sci 73:816-824. https://doi. org/10.1093/geronb/gbw044

Kastenbaum R, Derbin V, Sabatini P, Artt S (1972) “The ages of me": toward personal and interpersonal definitions of functional aging. Int J Aging Hum Dev 3:197-211. https://doi.org/10.2190/ TUJR-WTXK-866Q-8QU7

Kornadt AE, Hess TM, Voss P, Rothermund K (2018) Subjective age across the life span: a differentiated, longitudinal approach. J Gerontol B Psychol Sci Soc Sci 73:767-777. https://doi.org/10.1093/ geronb/gbw072 
Kornadt AE, Kessler EM, Wurm S, Bowen CE, Gabrian M, Klusmann V (2019) Views on aging: a life span perspective. Eur J Ageing. https://doi.org/10.1007/s10433-019-00535-9 (in press)

Kwak S, Kim H, Chey J, Youm Y (2018) Feeling how old I am: subjective age is associated with estimated brain age. Front Aging Neurosci. https://doi.org/10.3389/fnagi.2018.00168

Leist AK (2013) Social media use of older adults: a mini-review. Gerontology 59:378-384. https://doi.org/10.1159/000346818

Levy B (2009) Stereotype embodiment: a psychosocial approach to aging. Curr Dir Psychol Sci 18:332-336. https://doi.org/10.111 1/j.1467-8721.2009.01662.x

Macia E, DuBoz P, Montepare JM, Gueye L (2012) Age identity, selfrated health and life satisfaction among older adults in Dakar, Senegal. Eur J Ageing 9:243-253. https://doi.org/10.1007/s1043 3-012-0227-7

Montepare JM (1996) An assessment of adults' perceptions of their psychological, physical, and social ages. J Clin Geropsychol 2:117-128

Montepare JM (2009) Subjective age: toward a guiding lifespan framework. Int J Behav Dev 33:42-46. https://doi.org/10.1177/01650 25408095551

Montepare JM (2014) Same old, same old: persistent relations between women's body image and subjective age. Paper at the meeting of the Gerontological Society of America, Washington, DC

Montepare JM, Clements A (2001) Age schemas: Guides to processing information about the self. J Adult Dev 8:99-108. https://doi. org/10.1023/A:1026493818246

Montepare JM, Conte M (2014) Fashion attitudes and the aging self. Poster at the meeting of the American Psychological Association, Washington, DC

Pachana NA, Jetten J, Gustafsson L, Liddle J (2017) To be or not to be (an older driver): social identity theory and driving cessation in later life. Ageing Soc 37:1597-1608. https://doi.org/10.1017/ S0144686X16000507

Spuling, SM, Klusmann V, Bowen CE, Kornadt AE, Kessler EM (2019) The Uniqueness of subjective ageing: convergent and discriminant validity. Eur J Ageing. https://doi.org/10.1007/s1043 3-019-00529-7 (in press)
Stephan Y, Sutin AR, Terracciano A (2015) Subjective age and personality development: a 10-year study. J Pers 83:142-154. https ://doi.org/10.1111/jopy.12090

Stephan Y, Sutin AR, Caudroit J, Terracciano A (2016) Subjective age and changes in memory in older adults. J Gerontol B Psychol Sci Soc Sci 71:675-683. https://doi.org/10.1093/geronb/gbv010

Stephan Y, Sutin AR, Bayard S, Terracciano A (2017) Subjective age and sleep in middle-aged and older adults. Psychol Health 32:1140-1151. https://doi.org/10.1080/08870446.2017.1324971

Westerhof G, Wurm S (2015) Longitudinal research on subjective aging, health, and longevity: current evidence and new directions for research. Annu Rev Gerontol Geriatr 35:145-165. https://doi. org/10.1891/0198-8794.35.145

Westerhof GJ, Barrett AE, Steverink N (2003) Forever young? A comparison of age identities in the United States and Germany. Res Aging 25:366-383. https://doi.org/10.1177/016402750302500 4002

Westerhof G, Miche M, Brothers A, Barrett A, Diehl M, Montepare JM, Wurm S (2014) The influence of subjective aging on health and longevity: a meta-analysis of longitudinal data. Psychol Aging 29:793-802. https://doi.org/10.1037/a0038016

Wienert J, Kuhlmann T, Lippke S (2015) Direct effects of a domainspecific subjective age measure on self-reported physical activity: Is it more important how old you are or how old you feel? Health Psychol 2:131-139. https://doi.org/10.5114/hpr.2015.51450

Wienert J, Gellert P, Lippke S (2017) Physical activity across the life-span: does feeling physically younger help you to plan physical activities? J Health Psychol 22:324-335. https://doi. org/10.1177/1359105315603469

Wurm S, Schüz B (2015) Psychological theories on health and aging. In: Pachana NA (ed) Encyclopedia of geropsychology. Springer, Singapore, pp 1-10

Wurm S, Diehl M, Kornadt AE, Westerhof GJ, Wahl HW (2017) How do views on aging affect health outcomes in adulthood and late life? Explanations for an established connection. Dev Rev 46:2743. https://doi.org/10.1016/j.dr.2017.08.002

Publisher's Note Springer Nature remains neutral with regard to jurisdictional claims in published maps and institutional affiliations. 